\title{
Un enseignement par projet de la géotechnique
}

P. DELAGE

V. DE GENNARO

École nationale des ponts et chaussées

CERMES (Institut Navier)

6-8, avenue Blaise-Pascal

77455 Marne-la-Vallée

Cedex 2

\section{BERNHARDT \\ B. SIMON \\ Terrasol}

Immeuble Hélios

72, avenue Pasteur 93108 Montreuil Cedex

Un enseignement par projet a été mis au point en géotechnique à partir de l'étude de problèmes réels, modélisés par un code aux éléments finis géotechnique.

L'enseignement se déroule sur quatre séances et il est validé par une présentation devant jury avec remise d'un rapport. Ce contact plus proche avec la réalité de la pratique géotechnique permet de traiter divers aspects qui sont plus difficilement abordables dans le cadre de la pédagogie classique, La mise en évidence de la complexité des problèmes géotechniques ainsi que l'importance de l'expérience et du recours à l'intuition permettent de renforcer l'intérêt des étudiants pour la géotechnique.

Mots-clés : enseignement, projet, ouvrages, géotechnique, éléments finis, pédagogie.

\section{Project oriented geotechnical teaching} were modelled by using a geotechnical finite element code. The course is developed along 4 sessions and the validation is carried out by a final defence of the project in front of a committee. A written report is also asked. Thanks to this close contact with real geotechnical problems, various aspects that cannot easily be covered during conventional courses are considered. The complexity of geotechnical engineering is outlined. The necessary use of experience and intuition obviously strengthens the interest of students to geotechnical engineering.

Key words ; teaching, project, geotechnical engineering, geotechnical structures, finite elements, pedagogy. 


\section{Introduction}

Les vertus de l'enseignement par projet ne sont plus à démontrer. La réalisation d'un projet basé sur un cas réel à partir de données analogues à celles disponibles pour les ingénieurs de bureau d'études nécessite un investissement en temps important pour les étudiants et pour les enseignants, tant en termes de préparation que d'encadrement.

Un enseignement par projet de géotechnique a été mis en cuvre à l'ENPC dans le cadre de la voie d'approfondissement "Géotechnique » en deuxième année du cycle de formation d'ingénieur. Cet enseignement se situe dans la suite du cours d'initiation à la mécanique des sols du premier semestre (14 demi-journées). Il se déroule en parallèle avec le cours d'approfondissement géotechnique (14 demi-journées) où sont abordés les aspects relatifs au contexte géologique et géotechnique des ouvrages, aux mouvements de versants et au calcul d'ouvrages (fondations et soutènements).

La réalisation du projet géotechnique se déroule sur cinq séances de 3 heures, au sein d'un programme plus large (14 demi-journées) donné au tableau I pour l'année 2004-2005. Le tableau donne également le nombre d'encadrants à chaque séance, pour un effectif d'une trentaine d'élèves. Cet enseignement comprend des aspects expérimentaux de caractérisation géologique et géotechnique des sols au laboratoire (minéralogie, texture, œdomètre et triaxial) et in situ sur le terrain (pressiomètre, pénétromètre et scissomètre), quí ne seront pas détaillés ici. Les séances du projet sont intitulées P1 à P5 et sont espacées d'environ 3 semaines les unes des autres.
Le projet est basé sur l'utilisation d'un code aux éléments finis qui sert de support pour la modélisation de problèmes et d'ouvrages géotechniques réels, sur lesquels sont disponibles des données de terrain auxquelles les résultats obtenus seront confrontés. Il s'agit du code Plaxis (Brinkgreve et Vermeer, 1998), qui a démontré les qualités de convivialité et de robustesse qui permettent une assimilation rapide et l'obtention de résultats de qualité (distribution de contraintes, pressions interstitielles et déplacements) pour les problèmes aux limites proposés aux élèves. Il est à noter que d'autres codes géotechniques largement répandus, comme le code CESAR-LCPC (Humbert, 1989) par exemple, présentent également des qualités de convivialité qui leur permettraient de supporter ce type d'enseignement. 11 faut préciser enfin que les générations actuelles d'étudiants présentent de larges facilités d'assimilation de l'utilisation de toutes sortes de logiciels, ce qui facilite les choses.

Le nombre de séances dédiées au projet comprend quatre séances d'initiation et de suivi et une séance de soutenance devant un jury composé des enseignants. C'est un nombre relativement réduit pour un enseignement de ce type, ce qui requiert en conséquence une mobilisation personnelle effective de la part des étudiants en dehors des séances. Cependant, des séances de monitorat encadrées par des doctorants sont également mises en œuvre, les doctorants se rendant disponibles une à deux fois deux heures à heures fixes au cours de la semaine.

Pour une meilleure efficacité, il a finalement été choisi de présenter en parallèle lors des deux premières séances P1 et P2 (Tableau I) :

- une introduction aux lois de comportement; - la familiarisation au code Plaxis.

TABLEAUI Programme de l'enseignement de géotechnique appliquée intégrant le projet géotechnique (2005). Planning of the course of applied Geotechnics that includes the project oriented teaching (2005).

\begin{tabular}{|c|c|c|c|c|}
\hline$N^{\circ}$ & Date & Thème & & Lieu \\
\hline 1 & $9 / 3$ & \multicolumn{2}{|c|}{ Caractérisation des sols et des roches: minéralogie et texture I (CGI) } & Labo \\
\hline 2 & $16 / 3$ & \multicolumn{2}{|c|}{ Caractérisation des sols et des roches : minéralogie et texture II (CGI) } & Labo \\
\hline 3 & $23 / 3$ & \multicolumn{2}{|c|}{$\begin{array}{l}\text { P1: Initiation à la rhéologie des sols I: modèle Mohr-Coulomb (1 enseignant) } \\
\text { Introduction au logiciel Plaxis: présentation d'un exemple d'application (1 enseignant) } \\
\text { Question I (à rendre en P2) }\end{array}$} & $\begin{array}{l}\text { Salle } \\
\text { Salle }\end{array}$ \\
\hline 4 & $6 / 4$ & Réalisation d'essais en labo (CERMES) & Réalisation d'essais en place (Jossigny') & Labo \\
\hline 5 & $13 / 4$ & \multicolumn{2}{|c|}{$\begin{array}{l}\text { P2: Initiation à la rhéologie des sols: modèle Cam-Clay (1 eniseignant) } \\
\text { Introduction au logiciel Plaxis : exemple d'application à réaliser en salle (4 enseignants) } \\
\text { Présentation des projets aux groupes } \\
\text { Question II (à rendre en P3) }\end{array}$} & 2 salles info \\
\hline 6 & $20 / 4$ & Réalisation d'essais en labo (CERMES) & Réalisation d'essais en place (Jossigny) & Labo-Terrain \\
\hline 7 & $27 / 4$ & P3: Bilan avancement du projet (4 enseignants) & & 2 salles info \\
\hline 8 & $11 / 5$ & Réalisation d'essais en labo (CERMES) & Réalisation d'essais en place (Jossigny) & Labo-Terrain \\
\hline 9 & $18 / 5$ & P4: Bilan avancement du projet ( 4 enseignants) & & 2 salles info \\
\hline 10 & $25 / 5$ & Réalisation d'essais en labo (CERMES) & Réalisation d’essais en place (Jossigny) & Labo-Terrain \\
\hline 11 & $1 / 6$ & Réalisation d'essais en labo (CERMES) & Réalisation d'essais en place (Jossigny) & Labo-Terrain \\
\hline 12 & $8 / 6$ & Réalisation d'essais en labo (CERMES) & Réalisation d'essais en place (Jossigny) & Labo-Terrain \\
\hline 13 & $16 / 6$ & P5 Soutenance des projets & & 2 salles \\
\hline 14 & $22 / 6$ & $\begin{array}{l}\text { Synthèse et interprétation des donnẻes expérim } \\
\text { Remise du rapport de projet }\end{array}$ & tales & 1 salle \\
\hline
\end{tabular}

Centre de Géologie de l'Ingénieur, commun à l'École des mines, I'ENPC et l'université de Marne-la-Vallẻe

Site expérimental de Jossigny (77). 
Les séances P3 et P4 sont consacrées à l'encadrement et au suivi des projets, avec les inévitables rappels relatifs à une utilisation correcte et optimale du code et les dépannages rendus nécessaires par certaines utilisations erronées.

On présente plus en détail dans ce qui suit plusieurs aspects pédagogiques particuliers à cette forme d'enseignement.

\section{2.}

\section{Des lois de comportement}

Pour des raisons de temps, les lois de comportement ne peuvent pas être présentées suffisamment en détail lors du cours d'initiation à la mécanique des sols qui se déroule au premier semestre de la deuxième année. Quelques éléments sont cependant abordés lors des séances de TP qui accompagnent ce cours; ils comprennent la réalisation d'un essai triaxial simplifié sur un sable et l'analyse des courbes déviateur/déformation axiale et variation volumique/déformation axiale. On aborde ainsi les effets de la densité de l'échantillon. les réponses aux cycles de charge-décharge, les notions de limite élastique, de phase plastique avec déformations irréversibles, d'écrouissage et de contractance-dilatance.

L'objet de la première séance de projet (P1), où tous les élèves sont regroupés dans une même classe avec un enseignant unique, est double, avec une initiation aux lois de comportement et une première présentation du code utilisé :

- on vise à recadrer l'ensemble des éléments typiques du comportement des sols dans le cadre du modèle élastique linéaire parfaitement plastique obéissant au critère de rupture de Mohr-Coulomb, dont le principe est détaillé au tableau devant l'ensemble de la classe. Les écarts entre cette réponse idéale et la réponse du sol sont illustrés à, partir des courbes donnant les évolutions du déviateur et de la déformation volumique en fonction de la déformation axiale (Fig. 1). On rappelle en particulier que la réponse en compression élastique linéaire est indépendante de l'évolution du déviateur; le diagramme de la figure 1 montre également la correspondance entre l'apparition de la plasticité et la dilatance. On espère par cette comparaison relativiser la validité des applications calculées avec un modèle de comportement simplifié en faisant prendre conscience de ses limites propres, ainsi que des limites inhérentes à tout modèle;

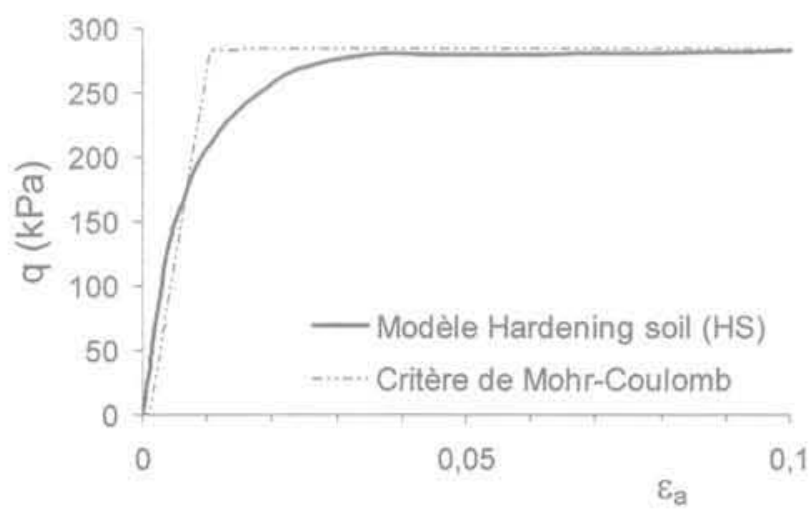

- on présente également le modèle élastoplastique appelé Hardening Soil, qui initie les étudiants aux modèles non linéaires intégrant l'écrouissage et une zone élastique fermée. Une première illustration de ce modèle est faite en comparaison avec le modèle de Mohr-Coulomb, afin d'illustrer en particulier le traitement de l'écrouissage. Le modèle Hardening Soil prévoit une élasticité linéaire caractérisée par un module d'Young $E_{u}$ et un coefficient de Poisson v $v_{u r}$ en déchargement-rechargement, avec un module sécant de référence à $50 \%$ de la rupture $E^{r e f}{ }_{50}=E_{u s} / 3$ intervenant dans la loi d'écrouissage hyperbolique; la rupture est définie par les mêmes caractéristiques que le modèle de Mohr-Coulomb (angle de frottement $\varphi^{\prime}$, angle de dilatance $\psi$ et cohésion c'). L'utilisation dans le modèle de Mohr-Coulomb d'un module d'Young $\mathrm{E}_{\text {ref }}=\mathrm{E}^{\mathrm{ref}}$ permet de bien corréler les rigidités initiales des courbes déviateur-déformation axiale d'un essai de cisaillement triaxial non drainé (Fig. 1). L'effet d'une zone élastique fermée est illustré par ailleurs par la modélisation d'un essai oedométrique et la mise en évidence d'un seuil de plasticité volumique;

- les caractéristiques principales du logiciel sont présentées avec la mise en œuvre en direct par l'enseignant par vidéoprojecteur d'un essai triaxial drainé qui est modélisé à l'aide des lois de comportement présentées ci-dessus.

Les étudiants ne manipulent donc pas directement le logiciel durant cette première séance. Leur premier contact se fait à l'occasion du travail personnel (Question I) qui leur est demandé pour la séance de projet suivante ( 3 semaines plus tard environ, voir tableau I). Il consiste à modéliser à l'aide du code, par groupe de 2 , un essai cedométrique ou un essai triaxial drainé/non drainé et à fournir pour la séance suivante un compterendu de cette modélisation par écrit. La première utilisation du code par les étudiants se fait donc hors encadrement, à l'aide d'un cas relativement simple et avec l'appui du guide fourni par le producteur du code, prêté aux élèves pour la durée du projet. Pour garantir le caractère personnel du travail rendu, des jeux de paramètres différents sont fournis à chaque équipe pour la modélisation des essais.

La séance P2 fait l'objet en première moitié de la présentation du modèle Cam-Clay et de ses dérivés en salle avec un enseignant. La seconde moitié de P2 a lieu, comme les séances P3 et P4, en salles informatique, la trentaine d'élèves étant répartie en deux groupes répartis à deux par ordinateur, avec deux enseignants par groupe.

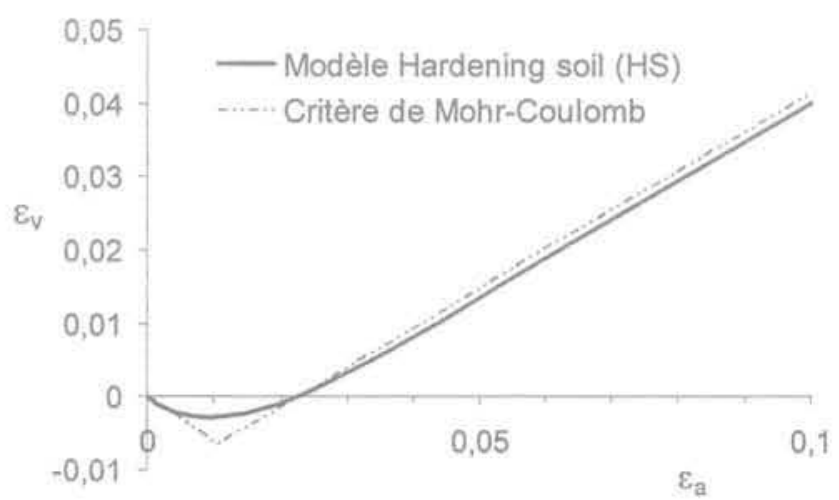

FG.1 Simulation d'un essai triaxial drainé sur sable selon différents modèles $\left(\mathrm{E}^{\text {ref }}{ }_{50}=\mathrm{E}_{\mathrm{ur}}=34 \mathrm{MPa} ; \mathrm{v}=0,3\right.$; $\varphi^{\prime}=35^{\circ} ; \psi=7^{\circ}$ et $\mathbf{c}^{\prime}=0$ ).

Modelling a triaxial drained test with different constitutive models. 


\section{Des projets proposés}

L'origine variée (bureau d'études et recherche) des intervenants permet de proposer divers types de projets :

- des projets plutôt de type conception, basés sur des problèmes rencontrés dans la pratique par les ingénieurs, pour lesquels le logiciel est utilisé pour modéliser et comparer diverses conceptions possibles. On a abordé des ouvrages aussi divers que des soutènements pour la réalisation de stationnements ou de murs de quai, des tunnels, des remblais avec des spécifications particulières en termes d'impacts sur d'autres structures (conduites...). Les déplacements résultant de la construction de l'ouvrage constituent souvent le critère de choix de l'option optimale. Il est à noter que la durée réduite de l'enseignement ne permet pas de chiffrer les coûts des projets et d'intégrer, comme dans la réalité, les aspects économiques. Cet aspect est cependant abordé globalement et qualitativement dans la définition des projets afin de ne pas déboucher sur des projets fantaisistes;

- des projets résultant d'actions de recherche ou de benchmarking qui permettent de disposer à la fois d'ouvrages réels instrumentés (déplacements, inclinomètres, contraintes dans les armatures ou ancrages) et de paramètres détaillés de sol résultant d'essais triaxiaux au laboratoire.

Une difficulté rencontrée régulièrement sur ces projets est précisément reliée à la détermination pertinente des paramètres à prendre en compte dans les modélisations: -il faut dans un premier temps que les étudiants perçoivent correctement la signification physique et le rôle des paramètres des lois de comportement, ce qui impose une bonne compréhension de ces lois (retours parfois nécessaires lors des séances P3 et P4 sur le contenu des séances $\mathrm{P} 1$ et $\mathrm{P} 2$, avec des questions résultant d'une assimilation incomplète de ces séances);

- la prise de conscience de l'incertitude et des approximations nécessaires sur les paramètres est perçue comme plutôt inconfortable par les étudiants qui, de fait, y sont confrontés pour la première fois; les paramètres de sol donnés pour les calculs lors des cours classiques - cohésion $c^{\prime}$, angle de frottement $\varphi^{\prime}$, coefficient de compression $\mathrm{C}_{c} \ldots$ - sont en effet fixes et uniques ! Il est à noter que la réalisation de calculs en déformations met en valeur l'importance des modules élastiques des sols, peu utilisés lors des cours classiques. Pour ce paramètre comme pour d'autres, le guide d'utilisation fournit un certain nombre d'ordres de grandeur et de variation de paramètres tout à fait intéressants dans la pratique (voir aussi Cassan 1987. 2005).

Les incertitudes et inconnues sur les paramètres correspondent à un problème couramment rencontré dans la pratique et l'approche par projet semble être une excellente façon de l'aborder concrètement.

L'expérience a montré qu'il était délicat de faire déduire par les étudiants les paramètres de lois de comportement à partir de paramètres pressiométriques obtenus in situ $\left(E_{M}\right.$ et $\left.p_{L}\right)$ et de la modélisation d'un essai pressiométrique (essai d'expansion de cavité, d'ailleurs traité dans le guide d'utilisation du code). La différence observée entre la modélisation et l'essai luimême (absence d'effet de forage en début de charge-

TABLEAU II Quelques projets proposés.

Some topics proposed.

Projet

Commentaire

Étude d'un essai de chargement de pieu

Étude d'une fondation mixte

Métro de Sao Paulo (station Luz)

Analyse du comportement d'une fondation de rembiai sur sol mou

Réinterprétation de désordres survenus durant la construction d'une écluse

Analyse des désordres d'un soutènement de quai en palplanches

Étude d'un barrage en remblai

Étude du mur cloué expérimental du CEBTP à Saint-Rémy

Analyse numérique de deux remblaís expérimentaux: Murro (Finlande) et Cubzac-les-Ponts (France)

Essai de chargement de pieu
Modélisation de l'essai de pieu foré de Roubaix (Frank et Zhao, 1982). Les paramètres de l'argile des Flandres sont tirés de Josseaume (1998) et on se réfere également à Frank (1999).

Modélisation d'un essai en vraie grandeur de fondation mixte, d'après Borel (2001).

Modélisation et analyse critique de plusieurs méthodes de construction: types de soutènement radial, phasage d'excavation, etc.

Exploitation des données des reconnaissances du terminal Transmanche d'Eurotunnel et application au projet d'un remblai théorique de hauteur donnée soumis à des contraintes de tassement différé.

Application de la méthode des éléments finis aux désordres survenus en 1961 lors des travaux de l'écluse de Ouistreham (Maisse et Desbazeille 1963).

Calage d'un modèle pour rendre compte des déplacements accidentels survenus 20 ans après la construction et extrapolation de ce modèle pour examiner les solutions de redressement.

Modélisation (écoulements et déformations) de barrages en remblai: - enrochement à noyau argileux;

- homogène à drain central.

Modélisation d'un mur cloué, calcul des efforts dans les clous et déformations (Plumelle, 1984; Schlosser et al., 1993).

Modélisation de la réponse de deux remblais expérimentaux. L'attention est portée, plus particulièrement, sur la consolidation à court et long terme de la fondation du remblai.

Modélisation d'un essai de chargement axial de pieu. Application des méthodes de dimensionnement classiques utilisées dans la pratique à partir des résultats du pressiomètre. 


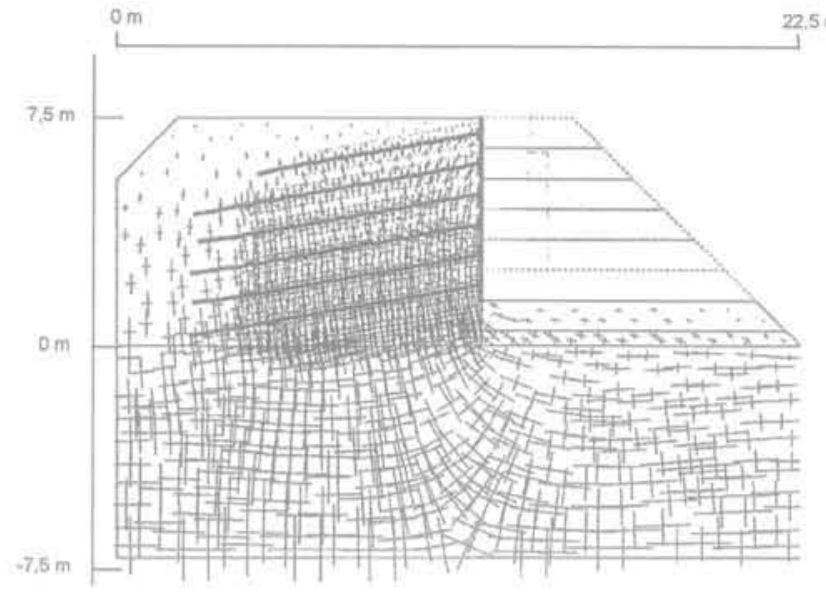

$22.5 \mathrm{~m}$

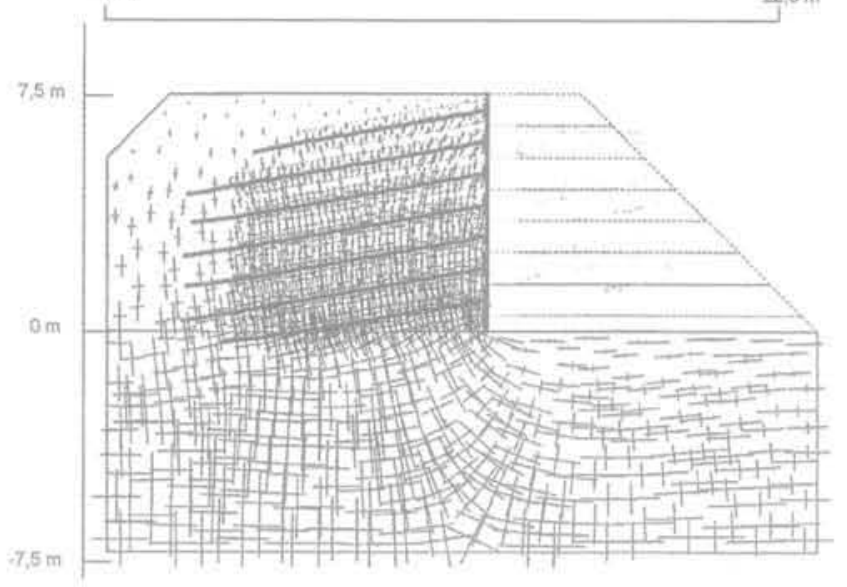

FIG.2 Modélisation du mur CEBTP de Saint-Rémy: variation des contraintes principales entre les phases d'excavation 6 et 8 .

Numerical modelling of the St Rémy nailed wall : changes in principal stresses distribution during excavation phases 6 and 8 .

ment, plastification moins nette au niveau de $\mathrm{p}_{\mathrm{L}}$ ), exploitable par un praticien plus expérimenté, a plutôt désorienté les élèves. D'où certaines difficultés quand les données de site proviennent uniquement d'essais de reconnaissance en place.

On a aussi observé une certaine impopularité auprès des étudiants des études paramétriques destinées à montrer la sensibilité du modèle aux valeurs de certains paramètres (effet de l'angle de dilatance sur les déformations, effet des modules élastiques sur les déformations...); ces approches sont perçues plus comme des exercices académiques que comme des projets proprement dits.

Autant que possible, les étudiants sont incités à comparer et recaler les résultats de modélisation obtenus par rapport aux résultats de méthodes de dimensionnement courantes enseignées classiquement. Cela permet de développer des analyses critiques relatives à la fois:

- à la validité et aux limitations de certaines méthodes classiques (hypothèses relatives à la mobilisation de la poussée et de la butée, par exemple);

- à la validité des hypothèses et des ordres de grandeur obtenus par les calculs aux éléments finis.

Il est en effet essentiel de développer chez les étudiants cette analyse critique des résultats de calculs aux éléments finis qui ne doivent pas être considérés comme vérité révélée du fait en particulier de l'esthétique de la présentation des résultats ischémas multicolores d'isovaleurs) ou de l'aspect complet et achevé d'une analyse qui fournit les champs de contraintes, de déformations et de pressions interstitielles.

A titre d'exemple, on donne avec quelques commentaires dans le tableau II un certain nombre de titres de projets proposés ces dernières années aux étudiants. La figure 2 présente les résultats obtenus par une équipe d'élèves en termes de contraintes principales calculées lors des phases 6 et 8 (phase finale) dans le cadre du projet d'étude du mur cloué expérimental de Saint-Rémy-lès-Chevreuse (Plumelle, 1984). Il s'agit d'un mur de $7 \mathrm{~m}$ de hauteur réalisé en sable de Fontainebleau (légèrement argileux) compacté de façon contrôlée qui a été réalisé par excavation de passes successives de $1 \mathrm{~m}$ avec à chaque passe mise en place de grillage, de béton projeté ( $8 \mathrm{~cm}$ d'épaisseur) et de clous inclinés de $10^{\circ}$.
Ce calcul bidimensionnel est basé sur l'utilisation intensive d'éléments d'interfaces entre les clous (ici des plaques) et le sol modélisé à l'aide du modèle Hardening Soil. Les paramètres ont été déduits de résultats d'essais triaxiaux (conduisant à un angle de frottement $\varphi^{\prime}=34,5^{\circ}$, de dilatance $\psi=5^{\circ}$ et à un module sécant de référence à $50 \%$ de la rupture $\mathrm{E}^{\text {ref }}{ }_{50}=30 \mathrm{MPa}$ ). Les calculs ont donné des valeurs de contraintes dans les clous très satisfaisantes par rapports aux mesures effectuées. Les déformations calculées se sont avérées très dépendantes des modules retenus pour le sol (sable de Fontainebleau), ce qui a donné lieu à une étude paramétrique. L'intérêt que présentent les diagrammes d'isovaleurs de contraintes principales (Fig. 2) pour une perception physique des redistributions de contraintes engendrées au cours des phases d'excavation est commenté aux élèves.

\section{4}

\section{Conclusion}

L'enseignement par projets dispensé en parallèle avec le cours de calcul des ouvrages géotechnique permet, via la modélisation de problèmes réels, de mettre les élèves au contact de problèmes géotechniques d'une façon plus approfondie que lors de cours classiques. Il ne s'agit pas d'un cours de modélisation numérique, le code utilisé constituant davantage un outil permettant d'aborder sur des cas réels des aspects aussi variés que:

- la difficulté du choix des paramètres représentatifs à partir de données parfois incertaines;

- le mode de fonctionnement d'une loi de comportement dépendant du type de sol rencontré;

- le fonctionnement des interfaces et des éléments de structures (éléments poutres, rotules plastiques, tirants d'ancrage...):

- l'analyse des résultats obtenus en termes de distributions de contraintes, de déformations engendrées en cours de construction, de champs de pression interstitielle et d'écoulements induits;

- la complexité du fonctionnement réel des ouvrages, en particulier en termes de couplages entre les contraintes et les pressions interstitielles; 
- l'optimisation des choix de conception du projet à partir des résultats de la modélisation.

L'analyse des questionnaires d'évaluation par les élèves a confirmé leur intérêt pour cette approche pédagogique qui semble avoir amélioré l'image qu'ils se faisaient de la géotechnique, en justifiant davantage, par la complexité rencontrée au cours du projet, certains choix ou approches géotechniques jugés a priorj rustiques ou peu évolués au regard des outils analytiques existants.
La gestion de la complexité abordée par ce type de cours a éveillé un intérêt particulier pour les étudiants souhaitant donner une part importante à l'intuition et à l'expérience dans leur pratique professionnelle. Cette vision a ainsi renforcé auprès des élèves l'image de la géotechnique et a contribué à attirer vers les métiers de la géotechnique des étudiants motivés et compétents. Plusieurs ont choisi une spécialisation en cette matière (master, thèse) ou ont intégré des bureaux d'ingénieur conseil spécialisés.

\section{Bibliographie}

Borel S. - Comportement et dimensionnement des fondations mixtes. Thèse de doctorat, École des ponts. Paris, 2001.

Cassan M. - Les essais in situ en mécanique des sols. Tome 1, réalisation et interprétation. Eyrolles, Paris, $2^{\circ}$ éd, 1987.

Cassan M. - Le pressiométre et la résistance au cisaillement des sols: cas particulie! des argiles saturées. ISP5 - Pressio 2005. Comptes-rendus du Symposium international 50 ans de pressiomètre, LCPC. Presses des Ponts, 2005, p. 699-714.

Brinkcreve R.B.J., Vermeer P.A. - PLAXIS V7.2 Finite element code for soil and rock analyses. Balkema, Rotterdam, 1998.
Frank R. - Calcul des fondations superficielles et profondes. Techniques de l'Ingénieur et Presses des Ponts, Paris, 1999.

Frank R., Zhao S.R. - Estimation par les paramètres pressiométriques de l'enfoncement sous charge axiale de pieux forés dans les sols fins. Bull. liaison Labo. P. et Ch. 119, 1982, p. 17 . 24

Humbert P. - CESAR-LCPC, un code général de calcul par éléments finis. Bull. liaison Labo. Ponts et Chaussées 160, 1989, p. $112-116$.

Josseaume H. - Propriétés mécaniques de
J'argile des Flandres à Dunkerque et à Calais. Revue française de qéotechnique 84, 1998, p. 3-26.

Malsse F. Desbazeille P. - Port de Caer Ouistreham: travaux d'amélioration et d'extension. Travaux 347, 1963, p. 509 520.

Plumelle C. - Expérimentation en vraie grandeur d'une paroi clouée. Revue française de géotechnique 40, 1984, p. $45-50$.

Schlosser F., Unterreiner P., Plumelle C. Validation des méthodes de calcul de clouage par les expérimentations du Projet national Clouterre. Revue française 